\section{ISOLATION OF STAPHYLOCOCCUS AUREUS FROM WOUND OF HANUMAN LANGUR}

\author{
M. Pal ${ }^{1}$ and P.B. Patil ${ }^{2}$ \\ ${ }^{1}$ Department of Veterinary Public Health, ${ }^{2}$ Department of Surgery \\ and Radiology, College of Veterinary Science, A.A.U., Anand, \\ Gujarat 388001, India \\ Email: 1 palmahendra2@rediffmail.com
}

Staphylococcus aureus, the principal causative agent of human and animals staphylococcosis, is a non-motile, non-sporulating, non-capsulated, Gram-positive organism (Pal, 1997). The pathogen is implicated in various clinical disorders of man as well as animals such as dermatitis, mastitis, gastroenteritis, arthritis, pneumonitis, endocarditis, osteomyelitis and meningitis (Pal \& Dube, 1981; Pal, 1997; Silverman et al., 1997). The perusal of available literature indicates scarcity of information on staphylococcal infection in monkeys from India (Arora, 2003). The present communication, therefore, describes the isolation of Staphylococcus aureus from the wound of an adult Hanuman Langur (Semnopithecus entellus).

An adult female Hanuman Langur (Semnopithecus entellus) was brought by the citizens of Anand to the Department of Surgery and Radiology for the purpose of treatment. The Himedia (India) swab was used to collect the specimen from the wound. The wound swab obtained in $1 \%$ glucose solution was immediately brought to the laboratory of Veterinary Public Health. The smears were prepared from the swab and examined under microscope after staining with Gram's stain. The specimen was cultured onto the plates of nutrient agar, blood agar and Sabouraud's dextrose agar with chloramphenicol. The inoculated plates were incubated at $37^{\circ} \mathrm{C}$ for microbial growth. The detailed identification of the organism was done as per the procedures recommended by Osbaldiston (1973). In vitro drug sensitivity of isolate was conducted against seven antimicrobial agents using the single disc diffusion test.

On clinical examination the female Hanuman Langur weighing $14 \mathrm{~kg}$ showed one deep wound on the right arm. The animal appeared dull, dehydrated, with severe ache in limb articulation and inability to move the arm. The body temperature was $36.6^{\circ} \mathrm{C}$, pulse $58 /$ minute and respiration $28 /$ minute. No other abnormality could be detected. There was no growth of fungi on mycological media. Interestingly, blood agar revealed circular, smooth, raised colonies with a type of haemolysis. Golden yellow coloured colonies were seen on nutrient agar. Microscopical examination of impression smear showed numerous gram-positive, non-capsulated, non-sporulated cocci arranged like a grape bunch. The isolate was positive for catalase and coagulase, and fermented lactose, mannitol and glucose. The organism was sensitive to ciprofloxacin, chloramphenicol, enrofloxacin, gentamicin and tetracycline but was resistant to ampicillin and penicillin. dog, deer, duck, geese, goat, horse, lion, monkey, pheasant, pig, poultry, rabbit, rhinoceros, tiger, wild civet and zebra ( $\mathrm{Pal}$, 1992; Blouse et al., 1996; Pal, 1997; Arora, 2003). The direct demonstration of $S$. aureus in the cutaneous lesion and its isolation in pure and heavy growth from the wound specimen indicated the pathogenic role of $S$. aureus in suppurative skin lesion of Hanuman Langur. Similar observations were reported by earlier workers who reported $S$. aureus from suppurative lesion in groin lesion of a rhesus monkey that died due to tuberculosis. S. aureus and Cryptococcus neoformans were recovered from the dermal lesions on the legs and abdominal areas in elephants (Arora, 2003).

As far as could be ascertained, the isolation of $S$. aureus from cutaneous lesion of a Hanuman Langur constitutes the first report from India.

\section{REFERENCES}

Arora, B.M. (2003). Indian Wildlife Disease and Disorders. Association of Indian Zoo and Wildlife Veterinarians, Barielly, U.P.

Blouse, L.E., R.M. Brockett, P.J. Homme and E.F. Jones (1996). Epizootic staphylococcal infections in subhuman primates after surgical operation. American Journal of Veterinary Research 37: 731-733.

Chakraborty, A. (1992). Incidences and etiopathology on the mortality of captive wild herbivores in Assam. Indian Journal of Veterinary Pathology 16:134-135.

Osbaldiston, G.W. (1973). Laboratory Procedures in Clinical Veterinary Bacteriology. University Press, Baltimore, London, Tokyo.

Pal, M. (1992). An epidemic of systemic staphylococcosis in White Leghorn Chicks. Veterinary Review 7: 24-25.

Pal, M. (1997). Zoonoses. R.M. Publishers, Delhi, India.

Pal, $M$ and G.D. Dube (1981). Isolation of $S$. aureus from canine mastitis. Agricultural Science Digest 1: 197-198.

Silverman, J., M.J. Loftus and M. Sharpless (1997). Mandibular osteomyelitis in a squirrel monkey. Contemporary Laboratory Animal Science 36: 94-96.

\section{ACKNOWLedgement}

We wish to thank the Dean of Veterinary Faculty, College of Veterinary Science, Anand Agricultural University, Anand, Gujarat for providing the necessary facilities to carry out the work and also to the staff of Veterinary Public Health and staff of Veterinary Surgery and Radiology, College of Veterinary Science, Anand for technical help.

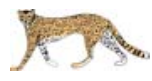

Staphylococcus aureus has been isolated from man, cat, cattle, 